\title{
Nursing home-acquired pneumonia: course and management in the emergency department
}

Syed Imran Ayaz ${ }^{1 *}$, Nadia Haque ${ }^{2}$, Claire Pearson', Patrick Medado', Duane Robinson', Robert Wahl', Marcus Zervos ${ }^{2}$ and Brian J O'Neil ${ }^{1}$

\begin{abstract}
Background: Pneumonia is among the foremost causes of hospitalization and mortality in patients residing in extended care facilities. Despite its prevalence, there is currently little literature focusing on the course and management of nursing home-acquired pneumonia (NHAP) in the emergency department (ED). Our objective was to investigate the ED presentation, course, management and outcomes in patients admitted through the ED with NHAP
\end{abstract}

Methods: A retrospective chart review of nursing home patients with a presumptive or final diagnosis of pneumonia admitted through the ED was performed at two large hospitals in Detroit, Michigan.

Results: A total of 296 patients were included in the study from 2002 to 2007 with a mean age of 81.1 years (SD \pm 10.95 ) and $55.4 \%$ females. Blood cultures were performed on $90.8 \%$ of patients in the ED; $17.8 \%$ of these revealed growths, but half of these were considered contaminants. Initial chest $x$-ray in the ED was read as possible pneumonia in $18.2 \%$ of patients; 73.9\% were started on antibiotics (ABX) in the ED. Mean hospital length of stay (LOS) was 10.75 days (SD \pm 9.35 ) and in-hospital mortality was $16.2 \%$. Time until first $A B X$ in univariate analysis was nearly significant $(p=0.053)$ for mortality prediction, and the appropriate versus inappropriate ABX (per the Infectious Diseases Society of America and American Thoracic Society guidelines) did not affect mortality. Patients treated with a single ABX had significantly increased LOS $(p=0.0089)$. There was poor correlation between LOS and time until first ABX as well as LOS and time until appropriate ABX with a correlation coefficient of $-0.048(p=0.42)$ and $-0.08(p=0.43)$, respectively.

Conclusions: In this data set of NHAP patients admitted through the ED, we found a surprisingly low prevalence of true-positive blood cultures, high incidence of antibiotic pre-treatment at nursing homes prior to admission, high hospital mortality and low immunization rates. There was a wide spectrum of pathogens grown in blood culture. Only two thirds of the patients had dyspnea at presentation, and less than half had either cough or fever. On physical examination, about one fourth had no clinical findings consistent with pneumonia. Further, less than one fifth of chest x-rays were interpreted as possible pneumonia.

Keywords: Nursing home; Pneumonia; Emergency department

\section{Background}

Pneumonia is among the foremost causes of hospitalization and mortality in patients residing in extended care facilities [1-8]. The microbes causing nursing home-acquired pneumonia (NHAP) are not well defined and include a wide spectrum from pathogens involved in community-acquired pneumonia (CAP)

\footnotetext{
* Correspondence: sayaz@med.wayne.edu

'Department of Emergency Medicine, Wayne State University School of Medicine, 4201 St. Antoine, 6G-UHC, Detroit, Ml 48201, USA Full list of author information is available at the end of the article
}

to those specific to nosocomial infections [9,10]. In 2005, the Infectious Diseases Society of America (IDSA) and American Thoracic Society (ATS), based upon data available to them, recommended empiric antibiotic treatment (ABX) guidelines for healthcare-acquired pneumonia (HCAP) including NHAP [11]. These treatment recommendations have not yet realized universal adherence, which may be due in part to the paucity of data available on NHAP $[12,13]$.

It has been well documented that the discrepancy in defining NHAP is an impediment toward a standardized

\section{实}

(c) 2014 Ayaz et al.; licensee Springer. This is an Open Access article distributed under the terms of the Creative Commons Attribution License (http://creativecommons.org/licenses/by/2.0), which permits unrestricted use, distribution, and reproduction in any medium, provided the original work is properly credited. 
approach to its management [12]. Absence of a precise scoring system for hospitalized patients with NHAP has also led to prognostic models that are inadequate in stratifying disease severity [12]. According to the 2005 ATS/ IDSA guidelines, a new or progressive infiltrate seen on chest X-ray plus compatible clinical signs, such as newonset fever with temperature greater than $38^{\circ} \mathrm{C}$, purulent sputum, leukocytosis or hypoxia, are essential features for the clinical diagnosis of HCAP, including NHAP [11].

Latest evidence suggests that transfer of nursing home patients with pneumonia to acute care facilities resulted in minor and insignificant improvement in mortality or morbidity when compared with patients who were treated at the nursing homes [14]. Once admitted, these patients are frequently managed as CAP in the hospital [1]. The management of NHAP continues to be controversial because of the scarcity of information on risk factors and diagnosis specific to this group of patients and lack of randomized controlled clinical trials that could help determine the best antimicrobial treatment regimen, length of treatment and rapid molecular techniques to ascertain etiological agents [15]. A previous study investigated the attitudes of physicians toward NHAP treatment guidelines and found that the core obstacles to implementation of the ATS/IDSA guidelines are apprehensions regarding the practicality of using the suggested regimens and absence of documented improved outcomes [13].

Advances in modern medicine have led to a rapid increase in the aging population with a subsequent increase in the number of elderly population requiring medical care [16]. An increase in the annual incidence of NHAP of up to 1.9 million cases is expected over the next 3 decades [14]. Despite its prevalence, there is currently little literature focusing on the course and management of NHAP in the ED. Similarly, little is known regarding the early predictors of mortality in these patients in the ED. Our objective was to investigate the ED presentation, course, management, outcomes, pathogens, appropriateness of $\mathrm{ABX}$, time to first
$\mathrm{ABX}$ administration and efficacy of $\mathrm{ABX}$ in relation to mortality and length of stay (LOS) as well as early predictors of mortality and LOS in patients admitted through the ED with NHAP. An understanding of the risk factors and outcomes of these patients is critical in the design of prevention and treatment strategies.

\section{Methods}

An Institutional Review Board (IRB)-approved, retrospective chart review was performed at two large tertiary care, academic teaching hospitals in Michigan. Henry Ford Hospital in Detroit is an 800-bed urban hospital with 93,000 emergency department visits annually. Similarly, Beaumont Hospital in Royal Oak is a 1000-bed community hospital with 115,000 emergency department visits per year.

Patients that resided in a nursing home or an extended care facility and who were admitted through the ED with a presumptive or final diagnosis of pneumonia were included in this retrospective chart review study. Patients were excluded from data abstraction if any of the following were noted during data abstraction: active cancer, tuberculosis, HIV, any immune suppression and hospitalization within the 3 weeks prior to the study visit. A standardized chart abstraction was performed collecting patient demographics, laboratory studies, radiographs, comorbidities, LOS, course of treatment, final outcome and ABX (pre- and post-ATS/IDSA guidelines). Our review of ABX included the time that the first antibiotics were ordered and administered and time until appropriate antibiotic administration (per the ATS/IDSA guidelines for HCAP). Additionally, we captured recommended antimicrobial therapy versus single antibiotic treatment on mortality and LOS. The included cases were retrospectively reviewed by trained abstractors using a standardized abstraction form. This study was performed in accordance with the previously published methods of Gilbert and colleagues [17].

All variables were entered into Statistical Package for Social Sciences (SPSS version $17.0^{\odot}$ SPSS Inc.) and

Table 1 Comorbidities and antibiotics started at the nursing home

\begin{tabular}{lclc}
\hline Comorbidities & \% of Patients & Nursing home antibiotics & \% of Patients \\
\hline Hypertension & 82.8 & Fluoroquinolones & 14.5 \\
Dementia & 55.1 & Amoxicillin and clavulanate & 3.3 \\
CHF & 42.2 & Co-trimoxazole & 3.1 \\
Stroke/TIA & 39.2 & Macrolides & 2.7 \\
COPD & 30.7 & Cephalosporins & 2.2 \\
Renal disease & 30.7 & Vancomycin & 2.0 \\
Diabetes mellitus & 26.4 & Anti-fungal & 2.0 \\
Alzheimer's & 22.3 & Piperacillin and tazobactam & 1.2 \\
Liver disease & 1.7 & Nitrofurantoin & 1 \\
\hline
\end{tabular}




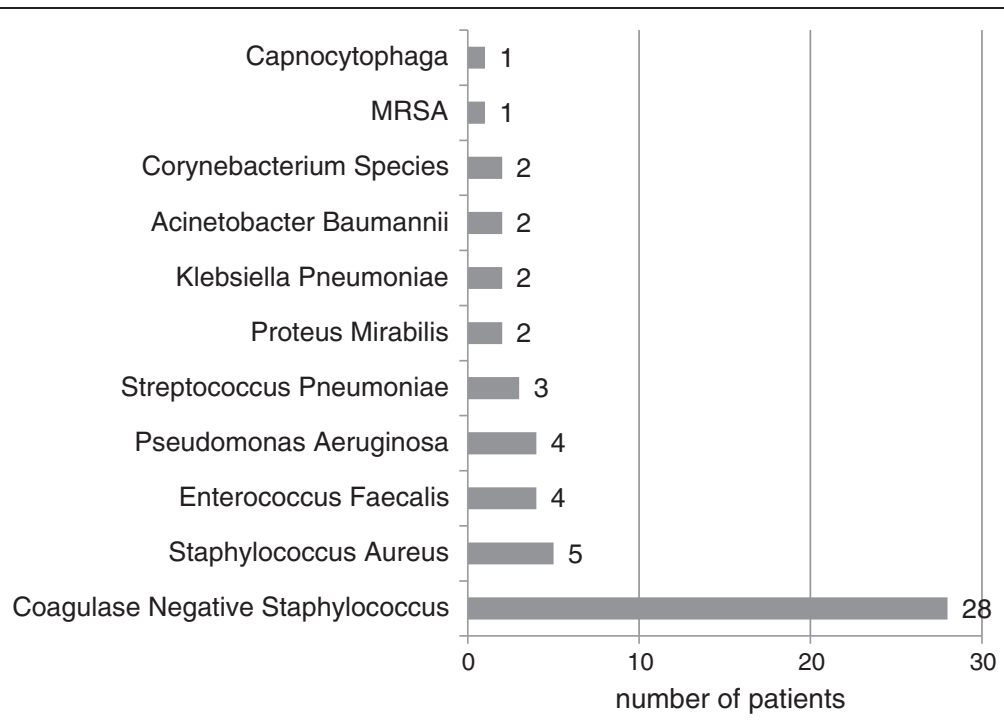

Figure 1 Pathogens revealed in blood culture performed in the ED.

descriptive analysis was performed. Means and standard deviations were calculated for continuous variables and frequencies determined for categorical variables. Categorical variables were examined using Pearson's chisquare and Fisher's exact test where appropriate. Continuous variables were analyzed using a Wilcoxon rank test and Spearman correlation. Step-down multiple-variable logistic regression analyses were performed to determine the strongest predictors of mortality.

\section{Results}

A total of 296 patients were included in the study from 2002 to 2007. Out of these 206 (69.6\%) patients were admitted to Beaumont Hospital and 90 (30.4\%) patients

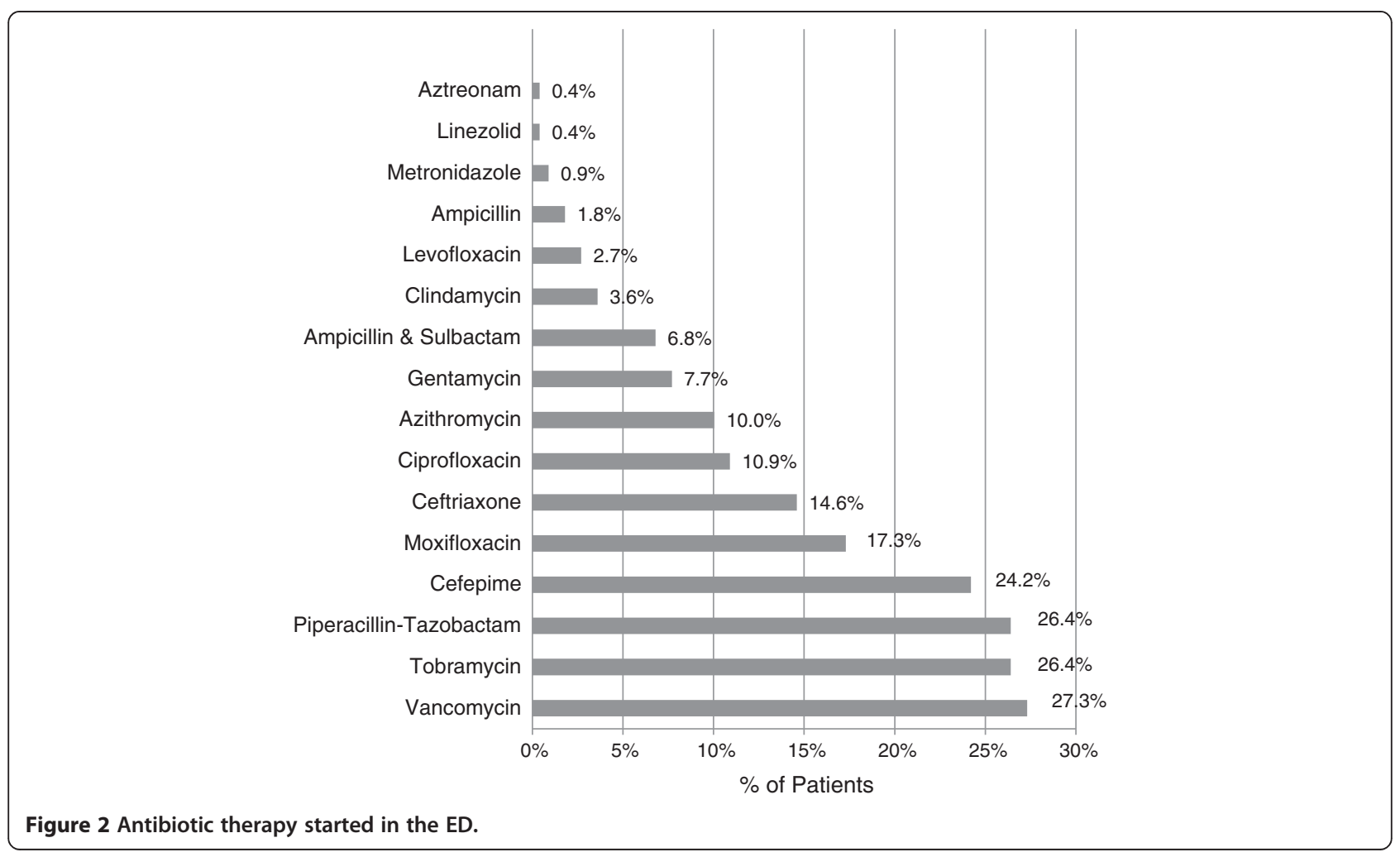


Table 2 Predictors of mortality in the ED

\begin{tabular}{|c|c|c|c|c|}
\hline & Parameter estimate & $P$ value & Odds ratio & 95\% Confidence interval \\
\hline Heart rate & 0.0117 & 0.028 & 1.012 & $1.001-1.022$ \\
\hline Temperature & -0.4184 & 0.0080 & 0.66 & $0.48-0.90$ \\
\hline Mental status change & 1.4054 & 0.0005 & 4.08 & $1.84-9.01$ \\
\hline Hemoglobin & -1.1004 & 0.0042 & 0.33 & $0.16-0.71$ \\
\hline Hematocrit & 0.2916 & 0.016 & 1.34 & $1.06-1.70$ \\
\hline BUN & 0.0124 & 0.037 & 1.012 & $1.001-1.024$ \\
\hline
\end{tabular}

were admitted to Henry Ford Hospital. The mean age of patients was 81.1 years $(\mathrm{SD} \pm 10.9)$. The gender distribution was 164 (55.4\%) females and $132(44.6 \%)$ males. A total of $237(80.1 \%)$ patients had pneumonia as one of the admission diagnoses. Other admission diagnoses were $20.9 \%$ sepsis, $17.2 \%$ UTI, $10.1 \%$ dehydration, $11.8 \%$ CHF, 9.5\% acute renal failure/renal insufficiency, 7.1\% acute respiratory failure, $6.8 \%$ COPD and $4.5 \%$ atrial fibrillation; $20.6 \%$ of the patients who had an admission diagnosis of pneumonia did not have a discharge diagnosis of pneumonia; $18.9 \%$ had received vaccinations in the past 1 year; $11.5 \%$ were vaccinated against influenza and $14.9 \%$ were vaccinated against pneumococcus. Eightytwo $(27.7 \%)$ patients had antibiotic therapy initiated in the nursing home (Table 1).

Blood cultures were performed on $90.87 \%$ of patients in the ED, $17.8 \%$ of whom revealed growth of organisms; however, $50 \%$ of these were considered to be contaminants. Contaminants were either defined as such in the medical record or were coagulase-negative staphylococcus in a non-immunocompromised patient (Figure 1). Sputum culture was obtained in $28 \%$ of the patients, $83.1 \%$ of which grew bacteria, none of which correlated to the identified blood pathogens. Two hundred nineteen (73.98\%) patients were given antibiotics in the ED (Figure 2). Cardiac enzymes were drawn in $75.7 \%$ of ED patients and were positive in $13.85 \%$. On initial ED ECG, 25.7\% had signs of ischemia and 18.2\% of the patients showed signs of pneumonia on their initial ED chest X-ray as interpreted by the final radiologist report.

Of the patients, $41.2 \%$ were admitted to the regular medical floor, $19.3 \%$ to the telemetry floor, $20.9 \%$ to step-down unit and $17.6 \%$ to the ICU; $12.16 \%$ of the patients were intubated for a mean duration of 9.28 days (SD \pm 12.2 ). Mean hospital LOS was 10.75 days (SD \pm 9.35), mean LOS in the ICU was 1.72 days (SD \pm 5.1 ), mean LOS in step-down/progressive care was 2.57 days $(\mathrm{SD} \pm 5.3)$, and mean LOS on regular medical floor with telemetry services was 6.2 days $(\mathrm{SD} \pm 7.3)$. Of the patients, $65.5 \%$ were discharged back to the nursing home, 3\% were discharged to a rehabilitation facility, 9.1\% were discharged under hospice care, and 3.7\% were discharged home; $16.2 \%$ of the patients expired during the initial hospitalization. Twenty-five percent of these were intubated, $25 \%$ of these were ICU-admits and $20.96 \%$ of were admitted to the step-down unit.

Of the patients, $41.6 \%$ were readmitted to the hospital within 1 year of their initial visit. Out of these, 54.5\% were discharged back to nursing homes, $3.3 \%$ to a

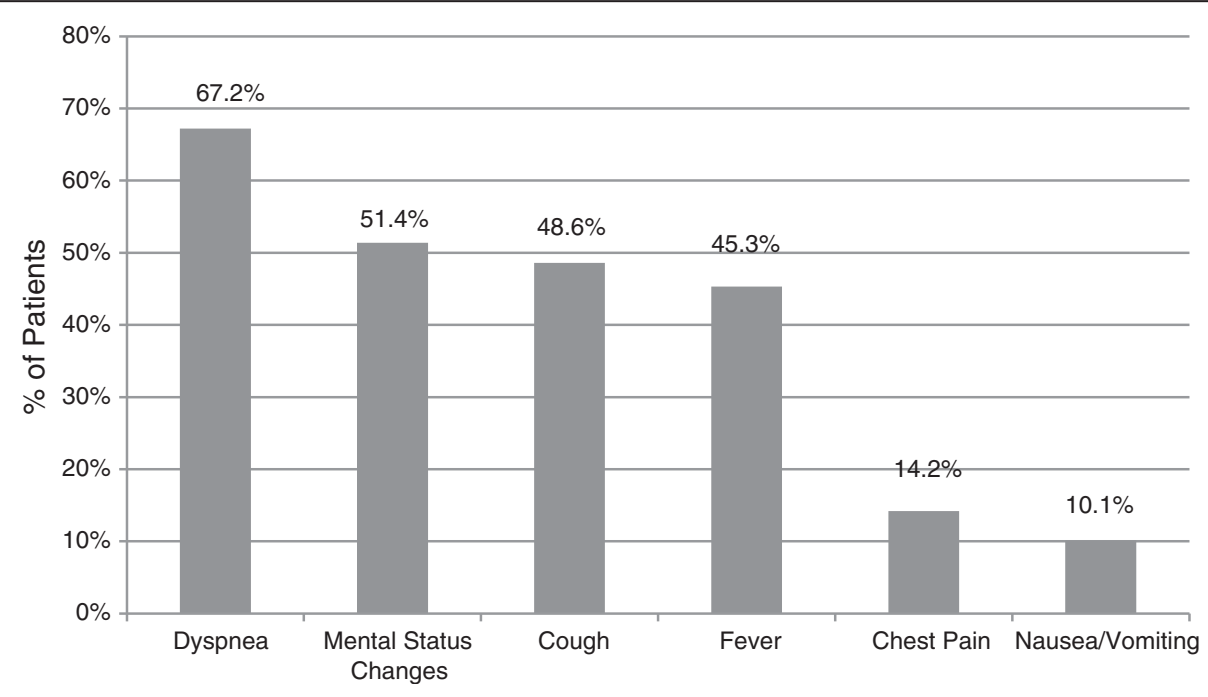

Figure 3 Symptoms on presentation to the ED. 


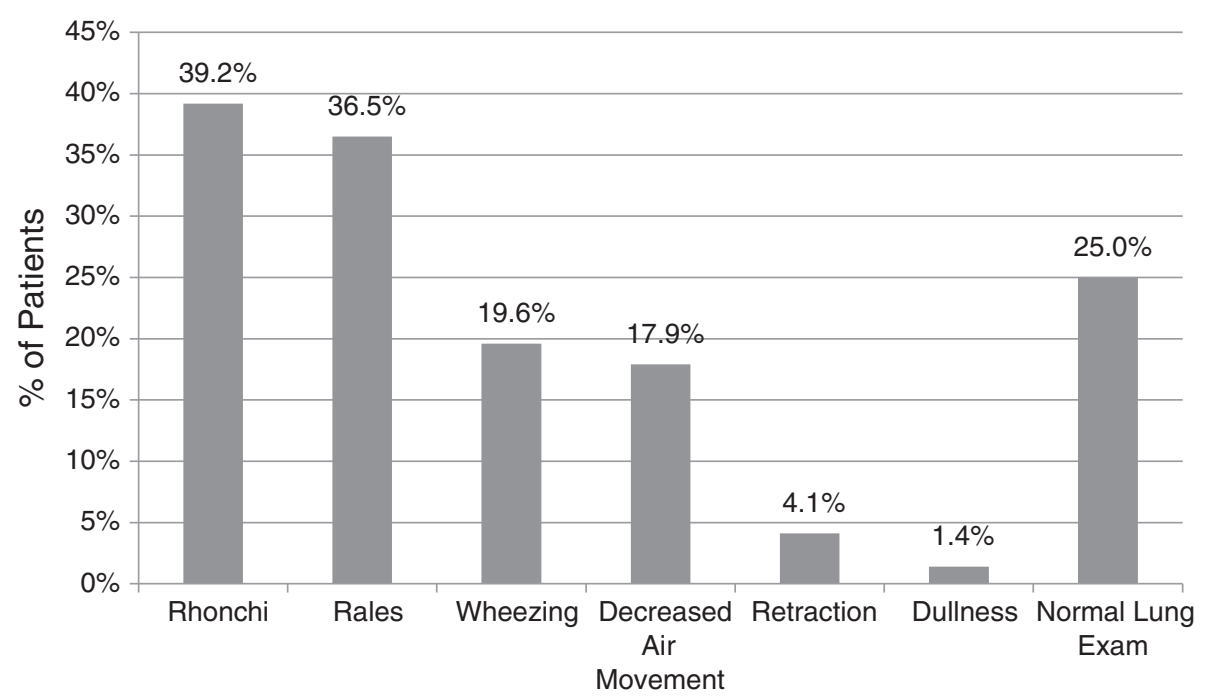

Figure 4 Physical examination findings on presentation to the ED.

rehabilitation facility, $6.5 \%$ under hospice care, and $14.6 \%$ home, and $8.1 \%$ of these patients expired during the 1-year follow-up re-admission to the hospital.

Comparing patients before and after the publication of the 2005 ATS/IDSA guidelines, NHAP was appropriately treated per the guidelines $59.4 \%$ versus $41.3 \%$, treated as CAP $26.1 \%$ versus $18.7 \%$ of the time and treated with a single antibiotic $46.4 \%$ versus $33.3 \%$, respectively. All comparisons were statistically significance with a $p$-value $<0.05$. Cefepime and tobramycin \pm vancomycin were utilized most often.

For mortality prediction, time until first antibiotic in univariate analysis was nearly significant, $p=0.053$, and the appropriate versus inappropriate antibiotic did not affect mortality. Regarding LOS, those treated with a single antibiotic had significantly increased LOS $(p=0.0089)$ and those treated with a CAP antibiotic had a shorter LOS $(p=0.049)$. Patients receiving clindamycin or piperacillin/ tazobactam had a longer LOS $(p=0.043$ and 0.038$)$, respectively. There was poor correlation between LOS and time until first $\mathrm{ABX}$ or LOS and time until appropriate

Table 3 Vital signs on presentation to the ED

\begin{tabular}{ll}
\hline & Mean $( \pm$ SD) \\
\hline Systolic blood pressure & $131.86 \mathrm{mmHg}(\mathrm{SD} \pm 30.14)$ \\
Diastolic blood pressure & $66.82 \mathrm{mmHg}(\mathrm{SD} \pm 16.12)$ \\
Heart rate & 92.38 beats per minute $(\mathrm{SD} \pm 22.92)$ \\
Respiratory rate & 24.74 breaths per minute $(\mathrm{SD} \pm 6.97)$ \\
Temperature & $37.35^{\circ} \mathrm{C}(\mathrm{SD} \pm 1.08)$ \\
Oxygen saturation & $94.15 \%(\mathrm{SD} \pm 6.085)$ \\
Initial GCS & $12.82(\mathrm{SD} \pm 2.96)$ \\
\hline
\end{tabular}

ABX with a correlation coefficient of $-0.048(p=0.42)$ and $-0.08(p=0.43)$, respectively.

Patients that died during initial hospitalization had a higher respiratory rate, glucose, blood urea nitrogen (BUN) and creatinine levels; lower GCS scores and hemoglobin level; more dyspnea on presentation; and increased incidence of mental status changes and intubations than those discharged alive. On step-down multivariable logistic regression analysis, increased heart rate, hematocrit and BUN, decreased temperature and hemoglobin, and presence of a mental status change were found to be the strongest predictors of death (Table 2). Patients who were intubated $(19.57 \pm 16.8$ vs. $9.31 \pm 6.6, p<0.0001)$ and febrile $(10.07 \pm 9.6$ vs. $11.58 \pm 9, p<0.012)$ had longer LOS. As age increases, LOS decreases (Spearman's correlation coefficient $-0.140, p=0.017)$; and as respiratory rate and temperature increase, LOS increases (Spearman's correlation coefficient 0.165 and $0.146, p=0.006$ and 0.015 , respectively).

Table 4 Laboratory values on presentation to the ED

\begin{tabular}{ll}
\hline & Mean $( \pm \mathrm{SD})$ \\
\hline WBC Count & $13.8 \times 10^{9} / \mathrm{L}(\mathrm{SD} \pm 12.3)$ \\
Hemoglobin Level & $11.4 \mathrm{mg} / \mathrm{dL}(\mathrm{SD} \pm 2.0)$, \\
Hematocrit & $34.9 \%(\mathrm{SD} \pm 6.4)$ \\
Platelet count & $287 \times 10^{9} / \mathrm{L}(\mathrm{SD} \pm 136.8)$ \\
Neutrophil count & $10.7 \times 10^{9} / \mathrm{L}(\mathrm{SD} \pm 6.8)$ \\
Lymphocyte count & $1.6 \times 10^{9} / \mathrm{L}(\mathrm{SD} \pm 5.0)$ \\
Blood glucose level & $146.39 \mathrm{mg} / \mathrm{dL}(\mathrm{SD} \pm 78.1)$ \\
BUN & $36.07 \mathrm{mg} / \mathrm{dL}(\mathrm{SD} \pm 26.4)$ \\
Serum sodium concentration & $138.17 \mathrm{mmol} / \mathrm{L}(\mathrm{SD} \pm 13.8)$ \\
Serum creatinine level & $1.7 \mathrm{mg} / \mathrm{dL}(\mathrm{SD} \pm 1.5)$ \\
\hline
\end{tabular}




\section{Discussion}

This data set represents one of the largest studies of nursing home patients with pneumonia presenting to the ED. We found a surprisingly low prevalence of true-positive blood cultures (9\%) and low yield and poor correlation in sputum cultures, high incidence of antibiotic pretreatment at nursing homes prior to admission (27.7\%), high hospital and 1-year mortality $(16.2 \%$ and $24.2 \%)$ and low immunization rates $(18.9 \%)$. There was a wide spectrum of pathogens grown in blood culture. Only two thirds of the patients had dyspnea as the presenting complaint and less than half had either cough or fever (Figure 3). On physical examination of the chest and lungs, about one fourth had no clinical findings consistent with pneumonia (Figure 4). Further, less than one fifth of chest $\mathrm{x}$-rays were interpreted as possible pneumonia. These findings coupled with few recorded fevers, hypoxia and elevated WBC counts make the ED diagnosis of pneumonia very difficult and therefore affects the management (Tables 3 and 4).

Since the 2005 ATS/IDSA guidelines, there has been a decline in the number of appropriately treated NHAP patients, as recommended by these guidelines, but also a decrease in the number of patients who were treated as having CAP or treated with a single antibiotic.

There was a correlation between time to first $\mathrm{ABX}$ and mortality. Those patients that received only a single ABX had a significantly increased LOS. Clindamycin, piperacillin/tazobactam or CAP-directed antibiotics also affected LOS; however, co-linearity with severity of disease may be present.

The best early ED predictors of in-hospital mortality are decreased mental status and anemia. Elevated temperature and signs of dehydration were also predictive of in-hospital mortality. Hospital length of stay was related to age, elevated temperature, intubation and respiratory rate.

\section{Conclusions}

In this data set of NHAP patients admitted through the $E D$, we found a surprisingly low prevalence of true-positive blood cultures and low yield and poor correlation in sputum cultures, high incidence of antibiotic pre-treatment at nursing homes prior to admission, high hospital mortality and low immunization rates. There was a wide spectrum of pathogens grown in blood culture. Only two thirds of the patients had dyspnea as the presenting complaint, and less than half had either cough or fever. On physical examination, about one fourth had no clinical findings consistent with pneumonia. Further, less than one fifth of chest $x$-rays were interpreted as possible pneumonia. In the light of these findings, we conclude that the patients presenting with pneumonia to the ED from nursing homes and longterm care facilities are a high-risk group with significant mortality and present unique diagnostic and treatment challenges to emergency physicians. Prospective, highquality randomized controlled trials investigating empiric antibiotic therapy to determine the effect of appropriate regimen on clinical outcomes are needed to support the implementation of the ATS/IDSA guidelines.

\section{Competing interests}

The authors declare that they have no competing interests.

\section{Authors' contributions}

SA performed the statistical analysis, interpreted the data and drafted the manuscript. NH contributed to data acquisition. CP interpreted the data and drafted the manuscript. PM and DR helped in data acquisition and analysis, and drafted the manuscript. RW drafted the manuscript. MZ and BON conceived the study, participated in its design and drafted the manuscript. All authors read and approved the final manuscript.

\section{Authors' information}

MZ is the Division Head of Infectious Diseases at Henry Ford Health System in Detroit, Michigan.

BON is the Chair of the Department of Emergency Medicine at Wayne State University and Specialist-in-Chief of Emergency Medicine at Detroit Medical Center in Detroit, Michigan.

\section{Acknowledgements}

This study was supported in part by Élan Pharmaceuticals. The authors declare that the sponsor had no role in the study design, in the collection, analysis and interpretation of data; in the writing of the manuscript; and in the decision to submit the manuscript for publication.

\section{Author details}

${ }^{1}$ Department of Emergency Medicine, Wayne State University School of Medicine, 4201 St. Antoine, 6G-UHC, Detroit, MI 48201, USA. ²Division of Infectious Diseases, Henry Ford Health System, 2799 West Grand Boulevard, Detroit, MI 48202, USA.

Received: 24 October 2013 Accepted: 20 April 2014

Published: 12 May 2014

\section{References}

1. Muder RR, Aghababian RV, Loeb MB, Solot JA, Higbee M: Nursing homeacquired pneumonia: an emergency department treatment algorithm. Curr Med Res Opin 2004, 20(8):1309-1320.

2. Quagliarello V, Ginter S, Han L, Van Ness P, Allore H, Tinetti M: Modifiable risk factors for nursing home-acquired pneumonia. Clin Infect Dis 2005, 40(1):1-6.

3. Carusone SC, Loeb M, Lohfeld L: A Clinical pathway for treating pneumonia in the nursing home: Part I: The nursing perspective. $J \mathrm{Am}$ Med Dir Assoc 2006, 7(5):271-278.

4. El-Solh AA, Niederman MS, Drinka P: Management of pneumonia in the nursing home. Chest 2010, 138(6):1480-1485.

5. Man SY, Graham CA, Chan SSW, Mak PSK, Yu AHY, Cheung CSK, Cheung PSY, Lui G, Lee N, Chan M, Ip M, Rainer TH: Disease severity prediction for nursing home-acquired pneumonia in the emergency department. Emerg Med J 2011, 28:1046-1050.

6. El-Solh AA, Alhajhusain A, Jaoude PA, Drinka P: Validity of severity scores in hospitalized patients with nursing home-acquired pneumonia. Chest 2010, 138(6):1371-1376.

7. Naughton BJ, Mylotte JM, Tayara A: Outcome of nursing home-acquired pneumonia: derivation and application of a practical model to predict 30 day mortality. J Am Geriatr Soc 2000, 48(10):1292-1299.

8. Mylotte JM: Nursing home-acquired pneumonia. Clin Infect Dis 2002, 35(10):1205-1211.

9. El-Solh AA: Nursing home-acquired pneumonia. Semin Respir Crit Care Med 2009, 30(1):16-25.

10. Polverino E, Dambrava P, Cillóniz C, Balasso V, Marcos MA, Esquinas C, Mensa J, Ewig S, Torres A: Nursing home-acquired pneumonia: a 10 year single-center experience. Thorax 2010, 65(4):354-359. 
11. American Thoracic Society: Guidelines for the management of adults with hospital-acquired, ventilator-associated, and healthcare-associated pneumonia. Am J Respir Crit Care Med 2005, 171:388-416.

12. El-Solh AA: Nursing home acquired pneumonia: approach to management. Curr Opin Infect Dis 2011, 24(2):148-151.

13. El-Solh AA, Alhajhusain A, Saliba RG, Drinka P: Physicians' attitudes toward guidelines for the treatment of hospitalized nursing home-acquired pneumonia. J Am Med Dir Assoc 2011, 12(4):270-276.

14. Dosa D: Should I hospitalize my resident with nursing home-acquired pneumonia? J Am Med Dir Assoc 2006, 7(Suppl3):74-80,

15. Mylotte JM: Nursing home-acquired pneumonia: update on treatment options. Drugs Aging 2006, 23(5):377-390.

16. Maruyama T, Gabazza EC, Morser J, Takagi T, D'Alessandro-Gabazza C, Hirohata S, Nakayama S, Ramirez AY, Fujiwara A, Naito M, Nishikubo K, Yuda $H$, Yoshida M, Takei Y, Taquchi O: Community-acquired pneumonia and nursing home-acquired pneumonia in the very elderly patients. Respir Med 2010, 104(4):584-592.

17. Gilbert EH, Lowenstein SR, Koziol-McLain J, Barta DC, Steiner J: Chart reviews in emergency medicine research: where are the methods? Ann Emerg Med 1996, 27(3):305-308.

doi:10.1186/1865-1380-7-19

Cite this article as: Ayaz et al:: Nursing home-acquired pneumonia: course and management in the emergency department. International Journal of Emergency Medicine 2014 7:19.

\section{Submit your manuscript to a SpringerOpen ${ }^{\circ}$ journal and benefit from:}

- Convenient online submission

- Rigorous peer review

- Immediate publication on acceptance

- Open access: articles freely available online

- High visibility within the field

- Retaining the copyright to your article 